\title{
110. Studies on Ventriculography
}

\section{Ist Report: Fundamental Studies on Emulsified Myodil Ventriculography using Ultrasonic Homoginizer}

\author{
Junichi Wakisaka, Shinken Kuramoto, Mitsuo Watanabe, \\ Kyoko Yoshimura, Towa RI and Kenji IwaI \\ 1st Department of Surgery, Kurume University School of Medicine
}

A new method of positive contrast ventriculography which used emulsified Pantopaque was reported by Portera-Sanchez et al. (1964) and Kitamura et al. (1967). They produced emulsion of Pantopaque with cerebrospinal fluid by manual shaking. Ultrasonic homogenizer was used in order to produce more dense and stable emulsion in our experimental studies. The following results were obtained:

1) The best condition of ultrasonic application was $180-220 \mathrm{~W}$ power, $25 \mathrm{Kc}$ frequency, $5 \mathrm{~min}$. application.

2) The manipulation of producing Myodil emulsion is very simple as follows: $10-20 \mathrm{ml}$ of spinal fluid are removed from the ventricular system into a glass cap containing 1-3 ml of Myodil, and it is dealed with ultrasonic homogenizer $5 \mathrm{~min}$.

3) A quality of emulsified Myodil is milky fluid of oil in water type emulsion, and its specific gravity was 1.010 , viscosity was $1.026, \mathrm{pH}$ was 6.33 , Jod contain was $2.7 \%$.

4) It was obtained fine contrast by emulsified Myodil on the $X$ ray examination using model ventricle. At present, we believe that emulsified Myodil is enough to use clinical ventriculography as a contrast medium.

\section{A New Method for the Direct Measurement of the Intracranial Pressure}

\author{
Kinjiro Iwata, Kazuyuki Watanabe, Takeshi KamiYa and Yoshimasa TanaKa \\ lst Dept. of Surgery, School of Medicine, Nagoya University \\ Isemi IGARASHI \\ Toyota Central Research \& Development Laboratories, Inc.
}

The measurement of intracranial pressure is one of the most important vital signs in the field of clinical neurology and neurosurgery.

We have reported here about our newly developed device to measure the 
intracranial pressure continuously and directly in the closed system with a pressure transducer using a semi-conductor strain gage which has been developed by Igarashi of Toyota Central R.D. Lab. Inc.

This pressure transducer is a small disc-type of $5 \mathrm{~mm}$ in diameter and $1.5 \mathrm{~mm}$ in thickness. Its out put power is $0.24 \mathrm{mV}$ to the pressure of $100 \mathrm{mmH}_{2} \mathrm{O}$, and it is recorded after amplifing. By this device, it is possible to measure the absolute value of intracranial pressure. The satisfactory results have been acquired in the animal and clinical measurement of intracranial pressure by this device. In clinical measurement, two kinds of plethysmograph were observed. One is the wave caused by respiration and the other is the wave caused by pulsation. The changes of these waves have important physiological significances. On the other hand, the absolute intracranial pressure also is changed very quickly and largely by cough, forced respiration, or the other changes of the condition of measuring subject. And in near future, it will become practical to measure the intracranial pressure by telemetering with using this device.

\title{
112. A Method of Radical Operation for Myelomeningocele with Upper Laminectomy
}

\author{
Shinken Kuramoto, Shigeyuki Takaki, Shigeyuki Baba, \\ Yoshiki YamashrTa and Ryoichi ONAKA \\ 1st Department of Surgery, Kurume University School of Medicine
}

We have recently experienced the two cases of meningocele and the four cases of myelomeningocele. We have operated the radical operation for all of these patients. For the two cases complicating the hydrocephalus we have operated the V-A shunt operation first and the radical operation next. We have gained the good results.

For the cases which are determined clearly as the myelomeningocele we have the operation of laminectomy, that is the method which separates the broken dura mater from the tissue enclosing. Our operation method has the following good points; it is easy to separate the tumor from its enclosing tissue, the rupture of dura mater is easily recognized, the damage of nerve system can be avoided and moreover the dura sutures can be operated easily and crtainly.

We consider it is the most important that the time of operation must not be behind time and the spinal cord coming off can be put into the dura mater speedily as possible as we can. 